\title{
Realistic Grand Canonical Monte Carlo Surface Simulation: Application to Ar(111)
}

\author{
Franck Celestini ${ }^{1,2}$, Daniele Passerone ${ }^{1,2}$, Furio Ercolessi ${ }^{1,2}$, and Erio Tosatti ${ }^{1,2,3}$ \\ (1) International School for Advanced Studies (SISSA-ISAS), I-34014 Trieste, Italy \\ (2) Istituto Nazionale di Fisica della Materia (INFM), Italy \\ (3) International Centre for Theoretical Physics (ICTP), I-34014 Trieste, Italy
}

(8 October 1997)

Preprint SISSA 133/97/CM/SS

To appear on Surface Science, 1998

\begin{abstract}
Most realistic, off-lattice surface simulations are done canonically-conserving particles. For some applications, however, such as studying the thermal behavior of rare gas solid surfaces, these constitute bad working conditions. Surface layer occupancies are believed to change with temperature, particularly at preroughening, and naturally call for a grand canonical approach, where particle number is controlled by a chemical potential. We report preliminary results of novel realistic grand canonical Monte Carlo simulations of the Lennard-Jones (LJ) fcc(111) surface, believed to represent a quantitative model of e.g. $\operatorname{Ar}(111)$. The results are successful and highly informative for temperatures up to roughly $0.8 T_{m}$, where clear precursor signals of preroughening are found. At higher temperatures, convergence to equilibrium is hampered by large fluctuations.

PACS numbers: 68.35.Rh, 68.35.Bs, 68.35.Md, 82.65.Dp
\end{abstract}

\section{Introduction}

Our quantitative understanding of thermal surface phenomena, including surface phase transitions, relies heavily on numerical simulations. Classical molecular dynamics (MD) and Monte Carlo (MC) simulations have played and play a crucial role, describing the surface either via lattice hamiltonians, or via continuous (offlattice) classical hamiltonians, or via continuous ab initio hamiltonians. The continuous hamiltonians are of course much more realistic, but they also have serious limitations. One of these limitations, which we address here, has traditionally been connected with strict particle conservation. It is desirable, in the study of many surface phenomena, to let the particle number fluctuate grand canonically. Lattice models permit that very naturally, but continuous models do not. In fact, there is so far very little experience of grand canonical surface studies conducted with continuous, off-lattice MD and MC simulation. In this paper we report on groundwork, plus some initial successes, in implementing a grand canonical Monte Carlo (GCMC) surface simulation based on a continuous classical hamiltonian, in particular the LennardJones fcc(111) surface, chosen as a model for rare gas surface.

Rare gas solid surfaces and films have provided an important testing ground for several surface phase transitions for over two decades. Surface melting, roughening, and recently preroughening have been studied and characterized at the free solid-vapor interface. Layering transitions of thin rare gas film on smooth substrates have given rise to a vast literature. The discovery of reentrant layering of rare gases on substrates has led to a debate [1] over a possible explanation in terms of preroughening, as suggested by RSOS models [2], versus a melting-solidification interplay [3] as argued from canonical simulations with a continuous potential. In real life, the true rare gas solid surface begins to develop diffusion phenomena, which are totally absent in lattice RSOS models, above roughly $(1 / 2) T_{m}$. However, the lattice models allow a much more thorough statistical mechanical understanding and classification of possible surface phases. In particular, they imply the possible existence of a preroughening (PR) transition, leading to a so called Disordered Ordered Flat (DOF) phase. At $T_{\mathrm{PR}}$, among other things, the surface occupancy exhibits a transition from close to a full monolayer $\left(T<T_{\mathrm{PR}}\right)$ to close to a half monolayer $\left(T>T_{\mathrm{PR}}\right)$. This kind of transition cannot be easily simulated with a fixed particle number. In fact it has been shown elsewhere [4,5] that a fixed particle number will lead, at and above $T_{\mathrm{PR}}$, to a kind of phase separation into two neighboring DOF phases. In other words, that surface will not even remain macroscopically homogeneous. Classical off-lattice GCMC [6] has so far be succesfully applied to simulate different systems such as capillary condensation in nanopores [7]. However difficulties appear when one tries to simulate a denser system [8] and the classical GCMC technique requires modifications. In the following we shall describe our own implementation.

\section{Grand canonical Monte Carlo surface simula- tion: technical details.}

Our implementation of GCMC is based on well known techniques [6], with modifications to improve the sam- 
pling efficiency in a system with a free surface. In particular, taking inspiration from an idea previously used for bulk liquids [8], we have restricted attempts to create or destroy particles to a region near the surface, where they are much more likely to be accepted.

We have considered three different types of Monte Carlo moves:

(a) small thermal displacements of individual atoms, with magnitudes $\delta r$ adjusted with $T$ in order to obtain an optimal acceptance rate of $50 \%$, and random direction;

(b) large lateral displacements of surface atoms;

(c) destruction of an existing particle, or creation of a new particle. Since deep in the bulk the acceptance rates will generally be exceedingly small, we restrict attempts to within a region of thickness $d=n a$, where $a$ is the spacing between two consecutive bulk lattice layers, and $n$ is typically 6 . This region is centered on the outer layer of the system simulated.

Moves of type (b) were thought at first to be important for establishing surface diffusivity. It was later seen, however, that this is not really so for the LJ surface. Therefore, they were eventually omitted. Clearly, only moves of type (c) are grand canonical. Thermal moves (a) must be by far the most frequent, for equilibration. We find that a large number of such moves is necessary, in the temperature range investigated, between a grand canonical move (c) and the next. We have taken the probability of attempting a move (c) to be $\alpha_{c}=\alpha_{d}=\alpha$ (respectively for creation and destruction), and that of attempting a move (a) to be $\alpha_{m}=1-\alpha$, with $\alpha$ very small, its actual value depending on the ratio between the number of atoms in the outer layers and the total number of atoms, but roughly of the order of $0.5 \times 10^{-3}$.

The acceptance probability of creation and destruction, $p_{c}$ and $p_{d}$ have then been finally normalized in the form [6]:

$$
\begin{gathered}
p_{c}=\min \left[1, \frac{V}{(N+1) \Lambda^{3}} \exp \left(\frac{\mu+\delta U}{k_{B} T}\right)\right] \\
p_{d}=\min \left[1, \frac{N \Lambda^{3}}{V} \exp \left(\frac{-\mu+\delta U}{k_{B} T}\right)\right]
\end{gathered}
$$

where $N$ is the number of atoms present in the creation/destruction region, $V$ its volume, $\Lambda=$ $\left[h^{2} /\left(2 \pi m k_{B} T\right)\right]^{1 / 2}$ is the thermal de Broglie wavelength, and $\delta U=U_{a}-U_{b}$ is the difference between the total energy $U_{a}$ after the trial move (creation or destruction) and the total energy $U_{b}$ before it. In order to satisfy detailed balance $N$ must be reajusted at each step of the simulation.

We simulated in this way the free fcc(111) solid-vapor interface of a rare gas, particularly Ar. The interatomic forces were described by a $(12,6)$ Lennard-Jones potential truncated at $2.5 \sigma$. Our system consisted of a 15 -layer slab, with periodic boundary conditions along the $x$ and $y$ direction in the interface plane. Three bottom atomic layers were kept frozen, while 12 layers of 480 atoms each were free to evolve. The lateral box size was rigid, but readjusted at each temperature according to the thermal expansion coefficient of this potential, obtained from separate bulk simulations [9]. We considered a grid of temperatures above $0.46 \epsilon$, as there is very little action below (the bulk melting temperature is $T_{B} \simeq 0.7 \epsilon$ ). For each temperature we first found the equilibrium value of the effective chemical potential $\mu_{\circ}(T)$. This was done by trial and error, starting from an arbitrary value, and changing it until the particle number remained as stationary as possible. With $\mu_{\circ}(T)$ so determined we made long equilibration runs looking for stabilization of both the total energy and the number of particles in the system. Generally half a million of Monte-Carlo (MC) steps (in the usual sense, i.e., one step attempting to move each particle on average once) were sufficient to reach reasonable equilibrium . Here we encountered our main problem, which is that this procedure is increasingly less convergent, and the resulting surface less stable, as $T$ increases. In practice, we found it impossible to stabilize the surface against exceeedingly large fluctuations above $T=0.56 \epsilon\left(\simeq 0.80 T_{m}\right)$. This constitutes an important drawback, because the interesting phase transitions of the surface are believed to lie just above this temperature, i.e., preroughening at $0.83 T_{m}$ and roughening at $0.95 T_{m}[5]$. On the other hand, a further reduction in the relative creation/destruction rate $\alpha$, necessary to obtain grand-canonical equilibrium at these higher temperatures, would quickly become too costly, as would the alternative possibility of creating/destructing atoms directly in the vapor phase. Below $0.80 T_{m}$, where our equilibration worked, typically 30 to 50 uncorrelated configurations were subsequently generated from another half a million MC steps run, and finally analysed.

\section{Results and discussion}

The typical $(x y)$-averaged density profile of the simulated grand canonical $\operatorname{Ar}(111)$ surface (really solid-vapor interface, but the vapor density is tiny) is shown in fig. 1. Layers are clearly visible, and the outermost ones are labeled 2 ("adatom layer"), 1 ("surface layer"), and 0 ("subsurface layer"). We conventionally associate an atom with a given layer $n$, if it falls within the bin $n$, as indicated. Taking the ratio of the number of atoms in layer $n, N_{n}$ to the number $N_{B}$ of atoms in a bulk layer, we obtain a conventional layer occupancy $o_{n}=N_{n} / N_{B}$. Occupancies of the three outermost layers are shown in fig. 2 2 as a function of $T$. At low temperatures, the layers 0 and 1 were almost full, with only a few percent of vacancies, with corresponding few adatoms in layer 2. As $T$ increased, layer 1 lost atoms very rapidly, reaching an occupancy $o_{1} \simeq 55 \%$ at $T=0.56 \epsilon$. 


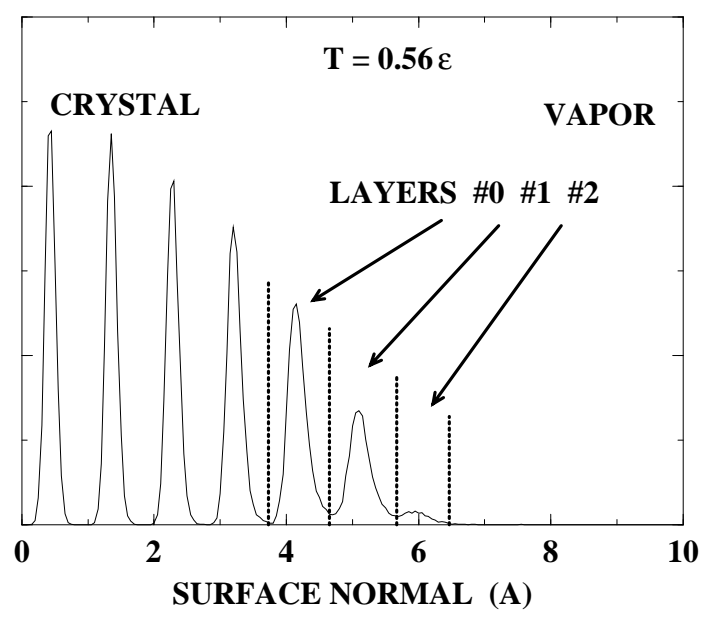

FIG. 1. (xy)-averaged density profile of the simulated Ar fcc(111) surface in grand canonical equilibrium at $T=0.56 \epsilon$, or $T=0.8 T_{m}$, plotted along the surface normal. The sub-monolayer coverage in layer 1 is the main new result, in comparison with previous canonical simulations.

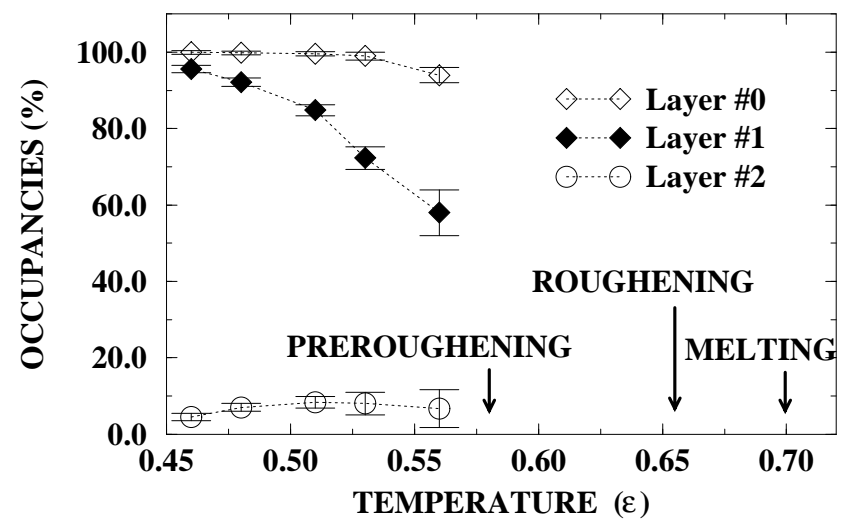

FIG. 2. Occupancies of the three outermost layers (fraction of particles contained in the bins indicated in fig. 1) as a function of temperature. Note the gradually decreasing occupancy of layer 1 , extrapolating to the preroughening value of $1 / 2$ close to $T=0.58 \epsilon$.

It is important at this point to compare the properties of this grand canonical Lennard-Jones (111) surface with those of exactly the same surface, simulated under identical physical conditions except for the canonical, particleconserving constraint. The results obtained in our earlier, very extensive MD simulations [5] were quite different. By starting with an integer number of layers, and at the very same $T=0.56 \epsilon$, for example, we had obtained canonically $o_{2} \simeq 100 \%, o_{1} \simeq 90 \%, o_{0} \simeq 10 \%$, against the present grand canonical values $o_{2} \simeq 94 \%, o_{1} \simeq 55 \%$, $o_{0} \simeq 8 \%$. Grand canonically, the "total surface population" $\left(o_{2}+o_{1}+o_{0}-1\right)$ appears to change continuously with temperature, going from nearly $100 \%$ at $0.65 T_{m}$ down to $57 \%$ at $0.8 T_{m}$. This kind of gradual change was not predicted by the less realistic solid-on-solid models [4, 10].

Extrapolating, these data strongly suggests that at $T \simeq 0.58 \epsilon$ the surface spontaneously tends to half occupancy, with about $8 \%$ adatoms and $6 \%$ subsurface vacancies. This temperature is in excellent agreement with the experimentally observed temperature for the onset of reentrant layering, $T=69 \mathrm{~K} \simeq 0.83 T_{m}$ [11. The coincidence with the preroughening temperature obtained from canonical simulations is perfect. The half occupancy also agrees with theoretical predictions and with solid-onsolid simulations of the disordered flat (DOF) state, realized at and above preroughening [10,12]. Therefore, the present grand canonical realistic simulation data strongly confirm the occurrence of preroughening and of a DOF phase on the free surface of $\operatorname{Ar}(111)$ at $T_{\mathrm{PR}}=0.83 T_{m}$.

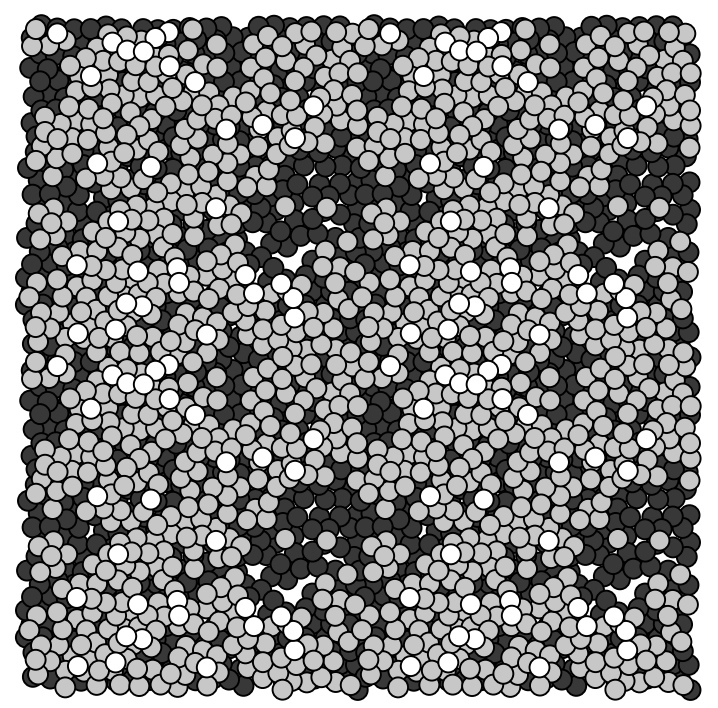

FIG. 3. Top view (snapshot) of the three outer layers of simulated grand canonical $\operatorname{Ar}(111)$ at $0.8 T_{m}$. This surface is nearly "disordered flat" (DOF). Atoms in layers 0, 1 and 2, identified through their $z$-coordinate as in fig. 1, are respectively black, grey and white. For convenience, four adjacent cells are shown. The nearly half occupancy of the surface layer (layer 1) is realized through large islands and large craters.

Fig. 3 presents a top snapshot of the grand canonical surface at $T=0.56 \epsilon$, the closest we can get to $T_{\mathrm{PR}}$. This is, we believe, the first available illustration of what a rare gas surface really looks like at preroughening. The main features to be noted are the large islands and craters in 
the first (gray) layer. We did not try yet to examine the height-height correlations of this surface, which would have been very interesting, particularly to check whether they are large as expected by our predicted divergence at $T_{\mathrm{PR}}$ (41. In order to do that properly, we would need finite-size scaling, which is computationally too demanding.

\section{Conclusions}

In conclusion, we have reported preliminary results on the microscopic nature of a rare gas solid-vapor interface, as obtained by means of a novel grand canonical Monte Carlo simulation. In spite of difficulties encountered with stabilizing the surface at higher temperatures, we have succeeded in describing what appears to be a well equilibrated surface in an interesting temperature range. We have obtained a clear indication that preroughening is indeed taking place under conditions very close to those where reentrant layering was observed on $\operatorname{Ar}(111)$, and where DOF phase separation was found by canonical MD [5]. We expect that, with the improvements which experience should soon bring about, grand canonical surface simulations should become the standard approach in the near future.

It is a pleasure to thank S. Prestipino, G. Santoro, and C. S. Jayanthi for constructive discussions. We acknowledge partial support from the European Commission under contracts ERBCHRXCT920062 and ERBCHRXCT930342, and INFM under PRA LOTUS. Work at SISSA by F. C. is under European Commission sponsorship, contract ERBCHBGCT940636.

[1] J. M. Phillips and J. Z. Larese, Phys. Rev. Lett. 75, 4330 (1995).

[2] P. B. Weichmann et al., Phys. Rev. Lett. 74, 418 (1995).

[3] J. M. Phillips et al., Phys. Rev. Lett. 71, 2971 (1993).

[4] S. Prestipino and E. Tosatti, Surf. Sci. 377-379, 509, (1997); and to be published (1997).

[5] S. Prestipino, C. S. Jayanthi, F. Ercolessi and E. Tosatti, to appear in Surf. Rev. Lett. (1997); C. S. Jayanthi, F. Ercolessi and E. Tosatti, to be published.

[6] L. A. Rowley, D. Nicholson and N. G. Parsonage, J. Comput. Phys. 26, 66 (1975).

[7] B. K. Peterson and K. E. Gubbins, Mol. Phys. 62, 215 (1987).

[8] M. Mezei, Mol. Phys. 40, 901 (1980).

[9] F. Ercolessi, unpublished.

[10] S. Prestipino, G. Santoro and E. Tosatti, Phys. Rev. Lett. 75, 4468 (1995).

[11] H. S. Youn and G. B. Hess, Phys. Rev. Lett. 64, 918 (1990); H.S. Youn, X.F. Meng and G.B. Hess, Phys. Rev.
B 48, 14556 (1993).

[12] M. den Nijs and K. Rommelse, Phys. Rev. B 40, 4709 (1989). 